\title{
Blockchain Technology and Healthcare Applications
}

\author{
Yu-Chun Tsai, MSN1,2; Nancy Hsao-Yen Chang, BSN3; Chin-Lung Kuo, MD, PhD ${ }^{4,5,6,7 *}$ \\ ' Office of Superintendent, St. Joseph's Hospital, Yunlin, Taiwan \\ ${ }^{2}$ Nursing Administration, Graduate Institute of Medical Sciences, Chang Jung Christian University, Tainan, Taiwan \\ ${ }^{3}$ University of Texas at Austin, Austin, Texas, USA \\ ${ }^{4}$ Department of Otolaryngology-Head and Neck Surgery, Taoyuan Armed Forces General Hospital, Taoyuan, Taiwan \\ ${ }^{5}$ Institute of Brain Science, National Yang-Ming University School of Medicine, Taipei, Taiwan \\ ${ }^{6}$ Department of Otolaryngology-Head and Neck Surgery, Tri-Service General Hospital, Taipei, Taiwan \\ ${ }^{7}$ Department of Otolaryngology-Head and Neck Surgery, National Defense Medical Center, Taipei, Taiwan
}

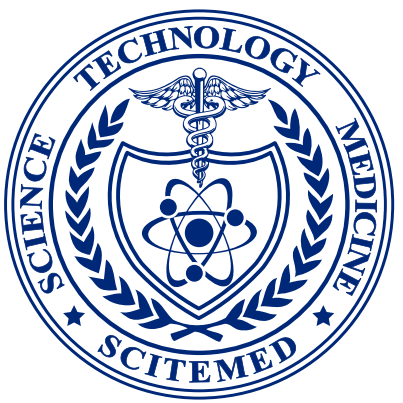

\section{INTRODUCTION}

A bockchain consists of a chain of blocks containing information. The chain is stored on nodes of computers that work collectively to maintain a shared ledger of information. The blocks record all transactions that take place in the system [1]. It is the basis of cryptocurrencies, of which the most famous is the Bitcoin [2-4]. Electronic cryptocurrencies are trusted and secure because encryption and validation are used to control the units and their transfer. Amendments can be made only according to a strict protocol.

The blockchain ledger is kept up to date on different nodes or computers. Bitcoin's blockchain ledger, for instance, prevents errors and double-spending, and keeps continuous track of transactions securely [5-7]. The records are on linked blocks and stored on an encrypted digital ledger, spread across a network of synchronised replicated databases. Users can only update the block they have access to. The system works without a central administrator, but since control is spread across the network, there are no chances of error. Because of its unprecedented security, a currency has been created that is not controlled by a central bank [8-10].

Events are recorded in each block that is authenticated using a protocol of operation. This mechanism ensures that information stored on the blockchain is trusted as reliable, since the network validates the blocks posted to the ledger as per the rules. The transaction data is thus replicated consistently across the network. Any mistake will be rejected as it will not be validated by the network [11-13].

The blockchain is said to be the most important creation since the advent of the Internet. Its design, based on a public, shared, tamperproof and trusted ledger, makes it open for a number of different applications. One such area is that of medical care [14-17].

\section{BLOCKCHAIN IN MEDICAL CARE}

The blockchain technology can be very helpful in integrating of health care information, which is currently scattered, with a range of service providers. Since it is a distributed network, blockchain-based systems can be useful for integration of several intermediaries in the medical care system. Such systems can be used in storing longitudinal patient histories that can be used by consumers, companies, and medical service providers [14,15]. One of its major advantages is interoperability among institutions and service providers. Blockchains can result in improved data integrity, decentralization and help in reducing transaction costs. They can also help in delivering precision medicine, improving patient care and outcomes, and the connecting medical records across a nation. This could be a boon in emergency situations as hospitals can easily access data rather than collect medical histories from individual patients $[16,18]$.

By combining these functions, blockchains can result in real-time patient monitoring and updating patient data by tracking different nodes using Internet of Things (IoT). Combined with a database, it can be used by medical care providers and a range of intermediaries. In this way, it allows for quick way of accessing patient records across service providers, thereby reducing costs, and improving collaboration between health institutions [1].

Blockchains can form the basis of Health Information Exchange (HIE) and the Integrating the Health care Enterprise (IHE) [19]. Further, by linking the system across functions such as insurance companies, financial and operational services, revenue cycles and supply chains, back-office data input and maintenance costs can be significantly reduced [20]. Data accuracy and security are added benefits. Countries could ultimately develop their nation-wide health information systems based on a seamless sharing of data. The benefits of integrating the medical network will also yield low cost solutions and virtual health systems in the long run. In summary, blockchain technology can be used in several key data-driven healthcare areas, including health care records, health claims, interoperability, patient access, and supply chains [21,22].

\section{Health Care Records}

Blockchains allow collection of health records of a patient's lifetime. This longitudinal data can be stored on blocks, which can yield invaluable insight not only with regard to individual patients but for various population segments. A distributed network can be used to improve care coordination among various services providers. For instance, a futuristic scenario has been described in which a technician can simply swipe patients' interactive wristbands to get their medical history. This would be of great help in medical emergencies, because as soon as the wristband is used, the patient's details are broadcast in real-time to the hospital and doctor, who use the blockchain to obtain access health care accounts with the details as recorded by the attending technician. Using loT, wearable sensors make medical care seamless no matter where the patient is. Furthermore, by combining health data from mobile applications with genomics can yield invaluable information about sub-populations who may be susceptible to a particular disease or who respond to certain kinds of treatment [23].

\section{Health Claims}

Another area for seamless integration is the area of health claims and insurance. Such claims, linked to a blockchain, can be easily and quickly processed, saving time and cost. Interoperability standards and application programming interfaces can provide an almost real-time claim settlement since patients' bank and health savings accounts would be linked through the blockchain $[1,24]$.

\section{Interoperability}

Health care records are traditionally disjointed and stored in silos of service providers. This is because Electronic Health Records (EHRs) were generated on an episode basis without taking into account linkages required for lifetime 
records or different service providers, and hence suffer from a lack of linkages, common architectures and standards [25]. There is also no system of transfer of patients' information among stakeholders and even to the patient. Thus, a patient's common clinical data cannot be updated in a central pool each time a medical service is provided. Interoperability is of great help that will help patients and medical service providers alike. Data interoperability allows systems that support population health initiatives, and the blockchain can be used to collect massive amounts of patient data to aid such large-scale initiatives [26].

\section{Patient Access}

Another benefit of an open ledger is that patients also gain access to their own medical records. This depends on health care data interoperability, which can be achieved after meeting regulatory and legal requirements. In addition, a health care blockchain can be linked to the latest medical research to help suggest the latest treatments for patients. Patients could thus be better informed and discuss the best treatment with their doctors based on research rather than accepted methods. The blockchain technology is thus useful for health care data interoperability by creating secure and trusted health record data, linking transactional data, and providing access to patients and recording their consent $[17,27]$.

\section{Supply Chains}

Blockchains can also link patients, service providers, and institutions to supply chains to monitor real-time tracking and execution. Consumers can get information about the quality, sources, and side effects of ingredients of the drugs they are prescribed $[15,16]$. This could ultimately lead to better productivity and quality control over the supply chain. A blockchain-enabled supply chain can track the goods right to the manufacturer, and also manage payments between sellers and buyers, thereby reducing overhead costs and by eliminating human error $[17,28]$.

\section{SUMMARY}

The blockchain technology has wide applications in health care. It may well lead to integrated health care data, which in turn with help countries to build their national health systems. There is little doubt that blockchains will be used in healthcare in a variety of ways. We believe that the blockchain will do to the medical system what the Internet did to media.

\section{ARTICLE INFORMATION}

*Correspondence: Chin-Lung Kuo, MD, PhD, Department of Otolaryngology, Taoyuan Armed Forces General Hospital, No.168, Zhongxing Rd., Longtan Dist, Taoyuan City 32551, Taiwan. E-mail: drkuochinlung@gmail.com

Received: Feb. 17, 2020; Accepted: May 29, 2020; Published:June 13, 2020

DOI: $10.24983 /$ scitemed.aohns.2020.00129

Ethics Approval and Consent to Participate: The study is in accordance with the ethical standards of the 1964 Helsinki declaration and its later amendments or comparable ethical standards.

Funding: This study was sponsored by grants from Medical Affairs Bureau Ministry of National Defense (MAB-107-099) and Taoyuan Armed Forces General Hospital (AFTYGH No. 10507, AFTYGH No. 10626, and AFTYGH No. 10734)

Conflict of Interest: The authors report no financial or other conflict of interest relevant to this article, which is the intellectual property of the authors.
Copyright (c) 2020 The Authors. This is an open-access article distributed under the terms of the Creative Commons Attribution 4.0 International License (CC-BY).

\section{REFERENCES}

1. Ahmad SS, Khan S, Kamal MA. What is blockchain technology and its significance in the current healthcare system? A brief insight. Curr Pharm Des 2019;25(12):1402-1408.

2. Bartolucci S, Caccioli F, Vivo P. A percolation model for the emergence of the Bitcoin Lightning Network. Sci Rep 2020;10(1):4488.

3. Tung JK, Nambudiri VE. Beyond Bitcoin: Potential applications of blockchain technology in dermatology. Br J Dermatol 2018;179(4):1013-1014.

4. Zimmer Z. Bitcoin and potosi silver: historical perspectives on cryptocurrency. Technol Cult 2017;58(2):307-334

5. Kim YB, Lee J, Park N, Choo J, Kim JH, Kim CH. When Bitcoin encounters information in an online forum: Using text mining to analyse user opinions and predict value fluctuation. PLoS One 2017;12(5):e0177630.

6. Extance A. Could Bitcoin technology help science? Nature 2017;552(7685):301-302.

7. Extance A. The future of cryptocurrencies: Bitcoin and beyond. Nature 2015;526(7571):21-23.

8. Chu J, Nadarajah S, Chan S. Statistical analysis of the exchange rate of Bitcoin. PLoS One 2015;10(7):e0133678.

9. Kondor D, Posfai M, Csabai I, Vattay G. Do the rich get richer? An empirical analysis of the Bitcoin transaction network. PLoS One 2014;9(2):e86197.

10. Kim T. The predecessors of Bitcoin and their implications for the prospect of virtual currencies. PLoS One 2014;10(4):e0123071.

11. Kohler S, Pizzol M. Life cycle assessment of Bitcoin mining. Environ Sci Technol 2019;53(23):13598-13606.

12. Dimitrova V, Fernandez-Martinez M, Sanchez-Granero MA, Trinidad Segovia JE. Some comments on Bitcoin market (in)efficiency. PLoS One 2019;14(7):e0219243.

13. Kristoufek $L$. What are the main drivers of the Bitcoin price? Evidence from wavelet coherence analysis. PLoS One 2015;10(4):e0123923.

14. Yoon HJ. Blockchain technology and healthcare. Healthc Inform Res 2019;25(2):59-60

15. Leeming G, Cunningham J, Ainsworth J. A ledger of me: Personalizing healthcare using blockchain technology. Front Med (Lausanne) 2019;6:171.

16. Hussien HM, Yasin SM, Udzir SNI, Zaidan AA, Zaidan BB. A systematic review for enabling of develop a blockchain technology in healthcare application: Taxonomy, substantially analysis, motivations, challenges, recommendations and future direction. J Med Syst 2019;43(10):320.

17. Agbo CC, Mahmoud QH, Eklund JM. Blockchain technology in healthcare: A systematic review. Healthcare (Basel) 2019;7(2)

18. Maxmen A. Al researchers embrace Bitcoin technology to share medical data. Nature 2018;555(7696):293-294

19. Mangesius P, Bachmann J, Healy T, Saboor S, Schabetsberger T. Blockchains in IHE-based networks. Stud Health Technol Inform 2018;251:27-30.

20. Lee HA, Kung HH, Udayasankaran JG, et al. An architecture and management platform for blockchain-based personal health record exchange: Development and usability study. J Med Internet Res 2020;22(6):e16748.

21. Hasselgren A, Kralevska K, Gligoroski D, Pedersen SA, Faxvaag A. Blockchain in healthcare and health sciences - A scoping review. Int J Med Inform 2020;134:104040.

22. Vazirani AA, O'Donoghue O, Brindley D, Meinert E. Blockchain vehicles for efficient Medica record management. NPJ Digit Med 2020;3:1.

23. Kim M, Yu S, Lee J, Park Y, Park Y. Design of secure protocol for cloud-assisted electronic health record system using blockchain. Sensors (Basel) 2020;20(10).

24. Park YR, Lee E, Na W, Park S, Lee Y, Lee JH. Is Blockchain technology suitable for managing personal health records? Mixed-methods study to test feasibility.J Med Internet Res 2019;21(2):e12533.

25. Dubovitskaya A, Xu Z, Ryu S, Schumacher M, Wang F. Secure and trustable electronic medical records sharing using blockchain. AMIA Annu Symp Proc 2017;2017:650-659.

26. Kuo TT, Kim HE, Ohno-Machado L. Blockchain distributed ledger technologies for biomedical and health care applications. J Am Med Inform Assoc 2017;24(6):1211-1220.

27. Roehrs A, da Costa CA, da Rosa Righi R. OmniPHR: A distributed architecture model to integrate personal health records. J Biomed Inform 2017;71:70-81.

28. Mackey TK, Kuo TT, Gummadi B, et al. 'Fit-for-purpose? - challenges and opportunities for applications of blockchain technology in the future of healthcare. BMC Med 2019;17(1):68. 